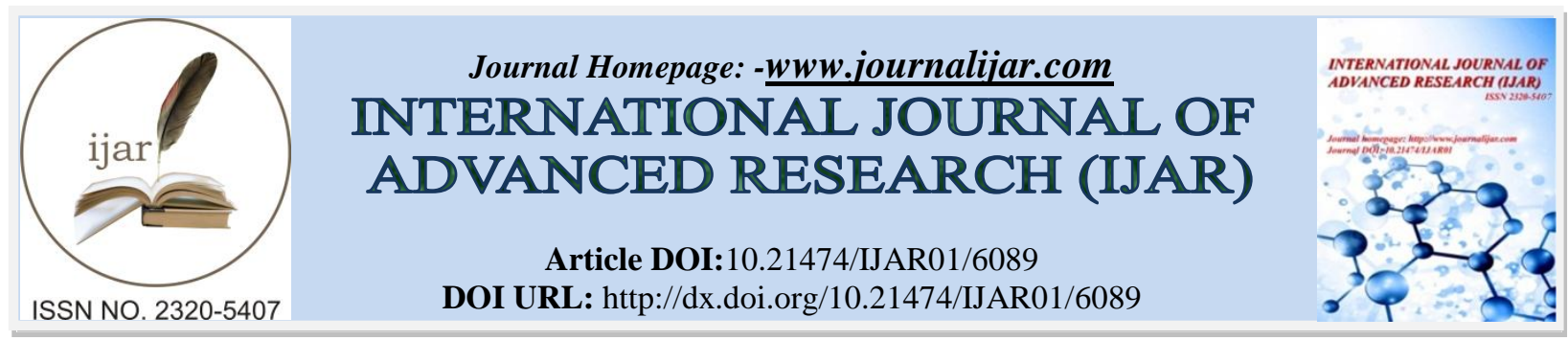

RESEARCH ARTICLE

\title{
DIVIDE ROW MINIMA AND SUBTRACT COLUMN MINIMA TECHNIQUE FOR SOLVING ASSIGNMENT PROBLEMS.
}

\author{
Mohammad Anwar Hossen and AfsanaAkther. \\ Department of Mathematics, Comilla University-3506, Bangladesh.
}

\section{Manuscript Info}

Manuscript History

Received: 18 October 2017

Final Accepted: 18 November 2017

Published: December 2017

Key words:-

Assignment Problem, Hungarian Assignment method (HA) and matrix ones assignment method (MOA), Linear Programming.

\begin{abstract}
Assignment problems deal with the question how to assign $\mathrm{n}$ objects to $\mathrm{m}$ other objects in an injective fashion in the best possible way. An assignment problem is completely specified by its two components the assignments, which represent the underlying combinatorial structure, and the objective function to be optimized, which models "the best possible way". The assignment problem refers to another special class of linear programming problem where the objective is to assign a number of resources to an equal number of activities on a one to one basis so as to minimize total costs of performing the tasks at hand or maximize total profit of allocation. In this paper we introduce a new technique to solve assignment problems namely,Divide Row Minima and Subtract Column Minima .For the validity and comparison study we consider an example and solved by using our technique and the existing Hungarian (HA) and matrix ones assignment method(MOA) and compare optimum result shown in graphically.
\end{abstract}

Copy Right, IJAR, 2017,. All rights reserved.

\section{Introduction:-}

In practical field we faced different types of problem which consists of jobs to machines , drivers to trucks, men to office etc. in which the assignees possess varying degree of efficiency, called as cost or effectiveness. The basic assumption of this type of problem is that one person can perform one job at a time. An assignment plan is optimal if it minimizes the total or maximizes the profit. This type of linear assignment problems can be solvd by the very well- known Hungarian method which was derive by the two mathematicians D.Konig and E.Egervary. Although the name "Assignment Problem" seems to have first appeared in 1952 paper by Votaw and Orden [1], what is generally recognized to be the beginning of the development of practical solution methods and variations on the classic assignment problem was the publication in 1952 of Kuhn's article on the Hungarin methods for it solution[2].Different methods have been presented for assignment problem and various articles have been published on see [3],[4],[5] for the history of this method.

In other words, the maximum size of a matching in a bipartite graph is equal to the minimum number of vertices needed to cover all edges. This result can be derived from that of Frobenius [1917], and also from the theorem of Menger [1927] but, as Konig detected, Menger's proof contains an essential hole in the induction basis. This induction basis is precisely the theorem proved by Konig. After the presentation by Konig of his theorem at the Budapest Mathematical and Physical Society on 26 March 1931, E. Egervary [1931] found a weighted version of Konig's theorem. It characterizes the maximum weight of a matching in a bipartite graph, and thus applies to the assignment nroblem. Egervarv's theorem and nroof method formed. in the 1950's. the imnulse for Kuhn to develop a 
new, fast method for the assignment problem, which he therefore baptized the Hungarian method. The first algorithm for the assignment problem might have been published by Easter field [1946], who described his motivation as follows:

Easter field seems to have worked without knowledge of the existing literature. He formulated and proved a theorem equivalent to Konig's theorem and he described a primal-dual type method for the assignment problem from which Egervary's result given above can be derived. Easter field's algorithm has running time. This is better than scanning all permutations, which takes time (n!).

In Dantzig [1951a] showed that the assignment problem can be formulated as a linear programming problem that automatically has an integer optimum solution. The reason is a theorem of Birkho [1946] stating that the convex hull of the permutation matrices is equal to the set of doubly stochastic matrices nonnegative matrices in which each row and column sum is equal to 1 . Therefore, minimizing a linear functional over the set of doubly stochastic matrices (which is a linear programming problem) gives a permutation matrix. The assignment problem has helped in gaining the insight that a finite algorithm need not be practical, and that there is a gap between exponential time and polynomial time. Also in other disciplines it was recognized that while the assignment problem is a finite problem, there is a complexity issue.

Lord [1952], Votaw and Orden [1952], and Dwyer [1954] (the 'method of optimal regions'). Von Neumann considered the complexity of the assignment problem. In a talk in the Princeton University Game Seminar on October 26, 1951, he showed that the assignment problem can be reduced to finding an optimum column strategy in a certain zero-sum two person game, and that it can be found by a method given by Brown and von Neumann [1950]. We give first the mathematical background. The basic combinatorial (non-simplex) method for the assignment problem is the Hungarian method. The method was developed by Kuhn [1955b,1956], based on the work of Egervary [1931], whence Kuhn introduced the name Hungarian method for it. On the origin of the Hungarian method"' Kuhn [1991] gave the following reminiscences from the time starting Summer 1953.See history [1],[2],[3],[4],[5] for this problem.

In this paper we introduce a new technique to solve assignment problems namely,Divide Row Minima and Subtract Column Minima on the basis of Hungarian (HA) and matrix ones assignment method(MOA). For the validity and comparison study we consider an example and solved by using our technique and the existing Hungarian (HA) and matrix ones assignment method (MOA).

Assignment Model in Mathematically :

Mathematically, the assignment problem can be expressed as follows:

Let $x_{i j}$ denote the assignment of facility $i$ to job $j$ such that

$x_{i j}=0$, if the $i$ th facility is not assigned to $j$ th job.

$x_{i j}=1$, if the $i$ th facility is assigned to $j$ th job.

\section{Optimize:}

$$
z=\sum_{i=1}^{n} \sum_{j=1}^{n} x_{i j} c_{i j}
$$

Subject to constraints $\sum_{\mathrm{j}=1}^{\mathrm{n}} \mathrm{x}_{\mathrm{ij}}=1, \mathrm{i}=1, \ldots \ldots \mathrm{n}$

$$
\begin{gathered}
\sum_{i=1}^{n} x_{i j}=1, j=1, \ldots \ldots . n \\
x_{i j}=0 \text { or } 1, i=1, \ldots \ldots . n, j=1, \ldots \ldots . n
\end{gathered}
$$$$
\}
$$

Where $c_{i j}$ is the cost or effectiveness of assigningith job to jthfacility and $x_{i j}$ is to be some positive integer or zero, and the only possible integer is one, so the condition of $x_{i j}=0$ or 1 , is automatically satisfied.

Associated to each assignment problem there is a matrix called cost or effectiveness matrix $\left[c_{i j}\right]$, where $c_{i j}$ is the cost of assigning ith job to jth facility. In this paper we call it assignment matrix, and represent it as follows: 


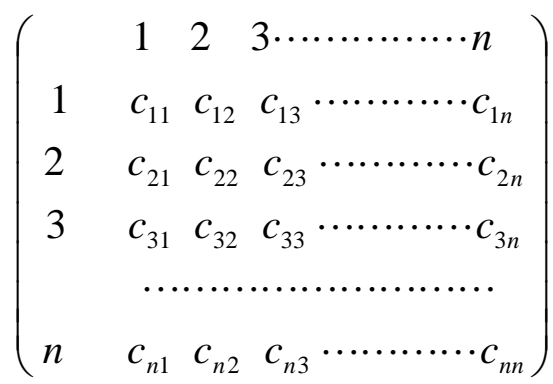

which is always a square matrix, thus each task can be assigned to only one machine. In fact any solution of this assignment problem will contain exactly $m$ non-zero positive individual allocations.

\section{Algorithm of Divide Row Minima and Subtract Column Minima method:-}

This is a new approach for solving assignment problem which is based on MOA-method and Hungarian-method. In this method we make assignment in terms of zeros. By a complete assignment we mean an assignment plan containing exactly $\mathrm{m}$ assigned independent zeros, zero in each row and zero in each column. Now, consider the assignment matrix where $c_{i j}$ is the cost or effectiveness of assigning ith job to jth machine.

Step-1:-Find the minimum cost of each row say $a_{i}$ and write it right hand side of this matrix. Divided each row by minimum $\operatorname{cost} a_{i}$

Step-2:-Find minimum cost of each column say $b_{j}$ and write it below of the matrix. Then subtract $b_{j}$ from jth column of the matrix.

Step-3:-Now make assignment in terms of zero. If there are some rows and columns without assignment ,then we cannot get optimum solution. Then we go to the next.

Step-4:-Draw the minimum cost of vertical and horizontal lines necessary to cover all the zeros in the reduced cost table obtained from Step 2 by adopting the following procedure:

1. Mark $(\sqrt{ })$ all rows that do not have assignments.

2. Mark $(\sqrt{ })$ all columns (not already marked) which have zeros in the marked rows.

3. Mark $(\sqrt{ })$ all rows (not already marked) that have assignments in marked columns.

4. Repeat steps 4(ii) and (iii) until no more rows or columns can be marked.

5. Draw straight lines through each unmarked row and each marked column. If the number of lines drawn (or total assignments) is equal to the number of rows (or columns) then the current solution is the optimal solution, otherwise go to Step 4.

Step-5: Select smallest cost of the of the reduced matrix not covered by the lines. Subtract this smallest cost from all not covered by a straight line and this same smallest cost is added to every cost including zeros lying at the intersection of any two lies . Other cost covered by lines remain unchanged. Again make assignment in terms of zeros.

Step-6: If optimum solution is not found ,repeat (4) \&(5) successively till an optimum solution is obtained.

\section{Numerical Example:-}

Example:- A project consist of four major jobs for which four contractors have submitted tenders. The tender amounts quoted in lacks of taka given in the matrix below: 


\begin{tabular}{|c|c|c|c|c|}
\hline \multirow{2}{*}{ Contractors } & \multicolumn{4}{|c|}{ Jobs } \\
\cline { 2 - 5 } & A & B & C & D \\
\hline 1 & 10 & 2 & 30 & 15 \\
\hline 2 & 16 & 22 & 28 & 12 \\
\hline 3 & 12 & 20 & 32 & 16 \\
\hline 4 & 9 & 26 & 34 & 10 \\
\hline
\end{tabular}

Find the assignment which minimize the total cost of the project, each contractor has to be assigned at least one job.

\section{Solve by using Divide Row minima and Subtract column Minimum method (proposed method):-}

\section{Step-1:-}

Find the minimum cost of each row say $a_{i}$ and write it right hand side of this matrix. Divided each row by minimum $\operatorname{cost} a_{i}$.

\begin{tabular}{|l|l|l|l|l|l|}
\hline \multirow{2}{*}{ Contractors } & Jobs & Min & \multirow{2}{*}{} \\
\cline { 2 - 5 } & A & B & C & D & 12 \\
\cline { 2 - 5 } & 5 & 1 & 15 & 7.5 & 10 \\
\hline 2 & 1.33 & 1.83 & 2.33 & 1 & 9 \\
\hline 3 & 1.2 & 2 & 3.2 & 1 & \\
\hline
\end{tabular}

Step-2:Now find the minimum cost of each column in assignment matrix ( say $b j$ ), and write it below that column. Then subtract this cost from each cost of jth column of the matrix

\begin{tabular}{|c|c|c|c|c|}
\hline \multirow{2}{*}{ Contractors } & \multicolumn{4}{|c|}{ Jobs } \\
\cline { 2 - 5 } & $\mathrm{A}$ & $\mathrm{B}$ & $\mathrm{C}$ & $\mathrm{D}$ \\
\hline 1 & 4 & 0 & 12.67 & 6.5 \\
\hline 2 & 0.33 & 0.83 & 0 & 0 \\
\hline 3 & 0.2 & 1 & 0.87 & 0 \\
\hline 4 & 0 & 1.89 & 0.5 & 0.78 \\
\hline Min & 1 & 2.33 & 1 \\
\hline
\end{tabular}

Step-3: Make initial assignment

Here we see that all zeros are either assigned or crossed out i.e. that is total assigned zero's is 4 which is equal to the no of rows or columns .And the optimum solution are $(1, B),(2, C),(2, B),(4, A)$.

\begin{tabular}{|c|c|c|c|c|}
\hline \multirow{2}{*}{ Contractors } & \multicolumn{4}{|c|}{ Jobs } \\
\cline { 2 - 5 } & $\mathrm{A}$ & $\mathrm{B}$ & $\mathrm{C}$ & $\mathrm{D}$ \\
\hline 1 & 4 & 0 & 12.67 & 6.5 \\
\hline 2 & 0.33 & 0.83 & 0 & 0 \\
\hline 3 & 0.2 & 1 & 0.87 & 0 \\
\hline 4 & 0 & 1.89 & 0.5 & 0.78 \\
\hline
\end{tabular}


The minimum cost $=2+28+10+9=49$

Solve the example using HA-method:

Step-1: Select the minimum cost of each row and subtract this cost from every cost in that row.

\begin{tabular}{|l|l|l|l|l|}
\hline \multirow{2}{*}{ Contractors } & \multicolumn{2}{|c|}{ Jobs } & C & D \\
\cline { 2 - 5 } & A & B & 28 & 13 \\
\hline 1 & 8 & 0 & 16 & 0 \\
\hline 2 & 4 & 10 & 22 & 0 \\
\hline 3 & 2 & 10 & 25 & 7 \\
\hline 4 & 0 & 17 & & 7 \\
\hline
\end{tabular}

Step-2: Select the minimum cost of each column and subtract this cost from every element in that column.

\begin{tabular}{|l|l|l|l|l|}
\hline \multirow{2}{*}{ Contractors } & \multicolumn{4}{|c|}{ Jobs } \\
\cline { 2 - 5 } & A & B & C & D \\
\hline 1 & 8 & 0 & 12 & 13 \\
\hline 2 & 4 & 10 & 0 & 0 \\
\hline 3 & 2 & 10 & 6 & 0 \\
\hline 4 & 0 & 17 & 9 & 7 \\
\hline
\end{tabular}

Step-3:- Make initial assignment

\begin{tabular}{|c|c|c|c|c|}
\hline \multirow[t]{2}{*}{ Contractors } & \multicolumn{4}{|c|}{ Jobs } \\
\hline & A & B & $\mathrm{C}$ & $\mathrm{D}$ \\
\hline 1 & 8 & $\overline{0}$ & 12 & 13 \\
\hline 2 & 4 & 10 & 0 & $y$ \\
\hline 3 & 2 & 10 & 6 & 0 \\
\hline 4 & 0 & 17 & 9 & 7 \\
\hline
\end{tabular}

Here we see that all zeros are either assigned or crossed out i.e. that is total assigned zero's is 4 which is equal to the no of rows or columns.And the optimum solution are $(1, \mathrm{~B}),(2, \mathrm{C}),(2, \mathrm{~B}),(4, \mathrm{~A})$.

The minimum cost $=2+28+10+9=49$

Solve the example using MOA-method:-

Step-1:-

Find the minimum cost of each row in the assignment matrix (say ai) and write it on the right hand side of the matrix Then divide each cost ofith row of the matrix by ai. These operations create ones to each rows, and the matrix reduces to following matrix.

\begin{tabular}{|c|c|c|c|c|c|}
\hline \multirow{2}{*}{ Contractors } & \multicolumn{4}{|c|}{ Jobs } & \multirow{2}{*}{ Min } \\
\cline { 2 - 5 } & $\mathrm{A}$ & $\mathrm{B}$ & $\mathrm{C}$ & $\mathrm{D}$ \\
& \multirow{2}{*}{2} & 7.5 \\
1 & 5 & 1 & 15 & 1 & 12 \\
\hline 2 & 1.33 & 1.83 & 2.33 & 1 & 10 \\
\hline 3 & 1.2 & 2 & 3.2 & 1.78 & 9 \\
\hline 4 & 1 & 2.89 & 3.75 & \\
\hline
\end{tabular}


Step-2:-Now find the minimum cost of each column in assignment matrix ( saybj), and write it below that column. Then divide each cost of jth column of the matrix by $b j$.

\begin{tabular}{|c|c|c|c|c|}
\hline \multirow{2}{*}{ Contractors } & \multicolumn{4}{|c|}{ Jobs } \\
\cline { 2 - 5 } & $\mathrm{A}$ & $\mathrm{B}$ & $\mathrm{C}$ & $\mathrm{D}$ \\
\hline 1 & 5 & 1 & 6.44 & 7.5 \\
\hline 2 & 1.33 & 1.83 & 1 & 1 \\
\hline 3 & 1.2 & 2 & 1.37 & 1 \\
\hline 4 & 1 & 2.89 & 1.62 & 1.78 \\
\hline Min & 1 & 1 & 2.33 & 1 \\
\hline
\end{tabular}

Step-3:- Make initial assignment

\begin{tabular}{|c|c|c|c|c|}
\hline \multirow{2}{*}{ Contractors } & \multicolumn{4}{|c|}{ Jobs } \\
\cline { 2 - 5 } & $\mathrm{A}$ & $\mathrm{B}$ & $\mathrm{C}$ & $\mathrm{D}$ \\
\hline 1 & 5 & 1 & 6.44 & 7.5 \\
\hline 2 & 1.33 & 1.83 & 1 & 1 \\
\hline 3 & 1.2 & 2 & 1.37 & 1 \\
\hline 4 & 1 & 2.89 & 1.62 & 1.78 \\
\hline
\end{tabular}

Here we see that all zeros are either assigned or crossed out i.e. that is total assigned zero's is 4 which is equal to the no of rows or columns .And the optimum solution are $(1, \mathrm{~B}),(2, \mathrm{C}),(2, \mathrm{~B}),(4, \mathrm{~A})$.

The minimum cost $=2+28+10+9=49$

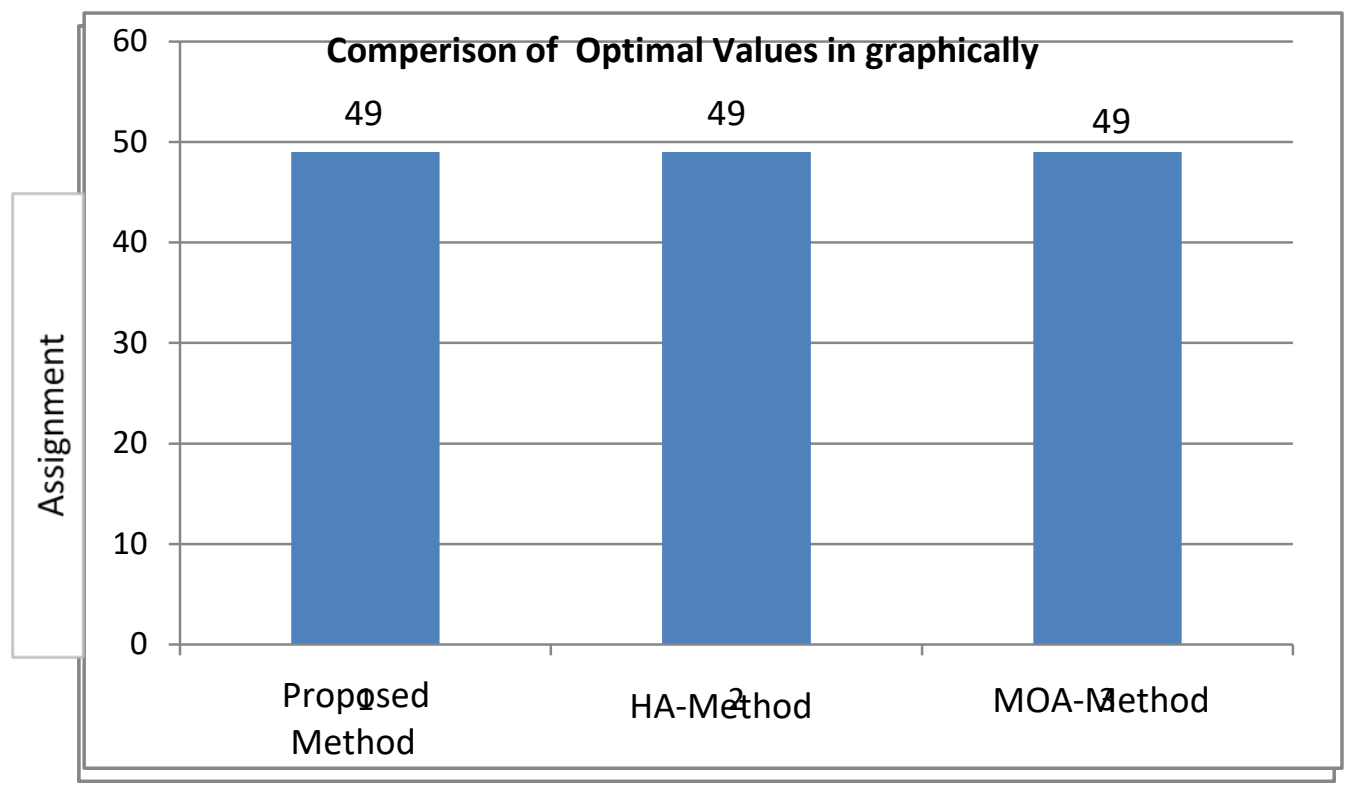

Table 1:-- Comparison of optimal values 


\begin{tabular}{|l|l|l|l|l|}
\hline Examples & $\begin{array}{l}\text { Proposed Method- } \\
(1)\end{array}$ & HA-Method & MOA-Method & Optimum \\
\hline I & 49 & 49 & 49 & 49 \\
\hline
\end{tabular}

\section{Conclusion:-}

In this paper, a new and simple technique was proposed for solving assignment problems. This method can be used for all kinds of assignment problems, whether maximize or minimize objective function. The new method is based on creating some zeros in the assignment matrix, and finds an assignment in terms of the zeros. This method offers significant advantages over similar methods. Also the comparison among three method have been shown in this paper graphically. Therefore this paper attempts to propose a technique for solving assignment problems which is differ from the preceding methods.

\section{References:-}

1. King, K. N., Java Programming from the Beginning, W.W. Norton \& Co., New York, 2000.

2. Kuhn, H. W. "The Hungarian method for the assignment problem", Naval Research LogisticsQuarterly2, 83-97.

3. Dantzig, B.G., "Application of the simplex method to a transportation problem", in Koopmans, T.C. (ed.), Activity Analysis and Production and Allocation, Willey, N.Y., 1951, 359-373.

4. Dantzig, B.G., Linear Programming and Extensions, Princeton University Press, Princeton.

5. D.F Votaw ,1952,A.Orden, The personal assignment problem ,Symposium on Linear Inequalities and Programming, SCOOP 10 US Air Force ,pp.155-163.

6. M. S. Bazarra, John J. Jarvis, Hanif D. Sherali, Linear programming and network flows, (2005).B. s. Goel, S. K. Mittal, Operations Research, Fifth Ed., (1982) 2405-2416.

7. Hamdy A. Taha, Operations Research, an introduction, 8th Ed. (2007).H. J. Zimmermann, Fuzzy set theory and its Applications, third Ed., kluwer Academic, Boston, 1996.

8. AnshumanSahu, RudrajitTapador, Solving the assignment problem usinggenetic algorithm and simulated annealing, IJAM, (2007).

9. Singh S.(2011) International Journal of Operation Research and International System,3(3),87-89.

10. Hung, M.S., and Rom, W.D., "Solving the assignment problem by relaxation", Operations Research, 28 (1980) 969-982.

11. Akgul, M., "A genuinely polynomial primal simplex algorithm for the assignment problem”, Working Paper IEOR 87-07, Bilikent University, Ankara, Turkey, 1987.Balinski, M.L., and Gomory, R.E., "A primal method for the assignment and transportation problems", Management Sciences, 10 (1964) 578-598.

12. Barr, R.S., Glover, F., and Klingman, D., "The alternating basis algorithm for assignment problems", Mathematical Programming, 13 (1977) 1-13.

13. Bertsekas, D., "A new algorithm for the assignment problem”, Mathematical Programming, 21 (1981) $152-171$.

14. Shweta sing and all. "A Comparative Analysis of Assignment Problem",IOSR Journal of Engineering(IOSREN),ISSN:2250-3021 Volume 2,Issue 8(August 2012),PP 01-15

15. HadiBasirzadeh, "One Assignment Method for Solving Assignment Problems",Applied Mathematical Science, Vol.6,2012 no.47,2345-2355. 